\title{
Efficient Fuzzy-Based System for the Diagnosis and Treatment of Tuberculosis (EFBSDTTB)
}

\author{
Angbera, Ature \\ Dept. Of Mathematics/ \\ Statistics/Computer \\ Science \\ University of Agriculture \\ Makurdi \\ Makurdi, Nigeria
}

\author{
Esiefarienrhe, Michael \\ Bukohwo \\ Dept. Of Mathematics/ \\ Statistics/Computer \\ Science \\ University of Agriculture \\ Makurdi \\ Makurdi, Nigeria
}

\author{
Agaji, Iorshase \\ Dept. Of Mathematics/ \\ Statistics/Computer \\ Science \\ University of Agriculture \\ Makurdi \\ Makurdi, Nigeria
}

\begin{abstract}
The aim of this study is to design a FuzzyBased Expert System for Tuberculosis diagnosis and Treatment. The designed system made use of General Hospital Adikpo, patient database. The system has 18 input fields and five outputs field. Input fields are Chest pain (CP), cough duration (CD), fever duration (FV), night sweats (NS), weight loss (WL), loss of appetite (LOA), change in bowel habits $(\mathrm{CBH})$, variations in mental behaviour (VMB), masses along the neck (MAN), draining sinus (DS), coma (seizure) (CO), stiff Neck (SN), headache (HD), abdominal Pain (AP), painful or uncomfortable urination (PU), hemopysis (coughing up blood) (CUB), fatigue (FA) and blood present in urine (BPU). The output fields refers to the class/group of tuberculosis disease in the patient. This system uses Mamdani inference method. The results obtained from designed system are compared with the data in the database and observed results of designed system are correct. The system was designed with Java (Jfuzzylogic), Microsoft visio (2013), mySql workbench, MySql database, JSP and XHML.
\end{abstract}

Keywords: Expert System, Diagnosis, Treatment, Inference component, Fuzzy Logic, Membership Function and Rules Blocks

\section{INTRODUCTION}

Computer technology tools help doctors to organize, store and retrieve relevant medical knowledge needed to understand the problematic cases and give them ideas about a proper diagnosis, prognosis and treatment decisions. There are huge data management tools available within health care systems, but analysis tools are not sufficient to discover hidden relationships amongst the data [9]. Expert Systems (ES) of an intelligent computer is based on interactive decision tool that uses facts and rules to solve real life problems, based on knowledge obtained from one or more of a human expert in a specific area.
In domain of disease like tuberculosis, which is one of the killer disease in developing countries. This disease has the following symptoms: fever, chest pain, coughing up blood, stiff neck, abdominal pain, variation in metal behavior, night sweat, urinating blood etc. Because of the many and uncertain risk factors in tuberculosis disease, sometimes the disease diagnosis is hard for experts.

Having so many symptoms to analyze to diagnose tuberculosis of a patient, the physician job is made very difficult. So, experts require an accurate tool that considering these numerous 
symptoms, a system that can diagnose and prescribe treatment (drugs) for tuberculosis in our health care centers is very important. Motivated by the need of such an important tool, in this study, we designed an expert system to diagnose tuberculosis disease and prescribe drugs for the patient. The designed expert system is based on Fuzzy Logic.

Fuzzy Logic is a form of multi-valued logic derived from fuzzy set theory to deal with

\section{REVIEW OF LITERATURES}

Tuberculosis (TB) is an infectious disease caused by mycobacteria, mainly Mycobacterium tuberculosis. It commonly attacks the lungs (pulmonary TB) but can also affect the central nervous system, the lymphatic system, the circulatory system, the genitourinary system, bones, joints and even the skin. Other mycobacteria such as Mycobacterium bovis, Mycobacterium africanum, Mycobacterium confetti and Mycobacterium microti, can also cause tuberculosis, but these species do not usually infect healthy adults, [11].

[5], proposed a fuzzy expert system for tuberculosis diagnosis which was developed for providing decision support platform to tuberculosis researchers, physicians, and other healthcare practitioners in tropical medicine. The combination of inadequate expertise and sometimes the complexity of medical practices exponentially increase the morbidity and mortality rates of tuberculosis patients. The task of arriving at an accurate medical diagnosis may sometimes become very complex and cumbersome. Fuzzy logic technology provides a simple way to arrive at a definite conclusion from vague, imprecise and ambiguous medical data. In order to achieve this, a study of the knowledge base system for tuberculosis was undertaken and a fuzzy expert system was developed using fuzzy logic technology [5]. Their system composed of four components which include the knowledge base, the fuzzification, the inference engine and defuzzification components. The fuzzy inference method employed in the research was the Root Sum Square (RSS). Triangular membership

www.ijcat.com approximate reasoning. It provides the means to represent and process the linguistic information and subjective attributes of the real world [4]. Most of the systems that are constructed based on fuzzy sets and logic have a common architecture. This fuzzy logic systems are based on a specific lifecycle model consisting of four characteristic stages namely, Fuzzifier component, Fuzzy Inference Engine, Fuzzy Rules and Defuzzifier component [15].

function was used to show the degree of participation of each input parameter and the defuzzification technique employed in this research is the Center of Gravity (CoG). The fuzzy expert system was designed based on clinical observations, medical diagnosis and the expert's knowledge. They selected 30 patients with tuberculosis and computed the results that were in the range of predefined limits by the domain experts [5]. [13], proposed a rule based Fuzzy Diagnostics and a decision support system which was intended to be used by pulmonary physicians, which will analyze the class of tuberculosis, by providing inputs (TB symptoms) into the system. In the formulation of fuzzy set system, the ranges of scores were classified in each symptom. The values or scores had undergone the process of fuzzification, which was also responsible for the threshold calculations that are needed by system for some reasoning, which are included in fuzzy relations. After processing the calculations, the resultant scores were graphed in a symmetrical manner. The graph will illustrate the scores and its corresponding membership values. After the graph process, the fuzzy logic sets were intersected and it determined a matrix format. The matrix illustrated that the symptoms are between the intersection points. The rules were determined by the scores that had undergone defuzzification process.

[1], designed an expert system for diseases diagnosis using Fuzzy set. In their approach, they used fuzzy set to diseases diagnosis, this was depending on opinion of 20 doctors. The result of the system shows the diagnosis of three types of 
respiratory diseases, which tuberculosis is one of them. The system used four symptoms, namely: $\mathrm{X}$ - ray, Respiratory rate (RR), Cough (CO) and Fever $(\mathrm{F})$ which were indicated as input of the fuzzy logic and the output was in a range of the risks and type of respiratory diseases.

[7], developed a diagnostic fuzzy cluster means system to help in diagnosis of Tuberculosis using a set of symptoms. The system which uses a set of clustered data set was more precise than the traditional system. The classification, verification and matching of symptoms to the seven groups of clusters was necessary especially in some complex scenarios. The model proposed allows for the classification and matching of cluster groups to TB symptoms.

[14], proposed the fuzzy Artificial Immune Recognition System (AIRS), for tuberculosis diagnosis detection. In designing the system, the Fuzzy Logic Controller was applied, which converts the continuous inputs into fuzzy sets. Ten features of tuberculosis diagnosis were defined for the fuzzy input. Asthma is a chronic inflammatory lung disease. An automated system

\subsection{TB Disease Treatment Regimens}

In a study by [3], there are four basic treatment regimens recommended for treating patients with TB disease caused by organisms that are known or presumed to be susceptible to Isoniazid (INH), Rifampin (RIF), Pyrazinamide (PZA), and

\section{METHODOLOGY}

\subsection{The Proposed System}

The system is designed to aid in the diagnosis and treatment of TB in our settings. The success of any Fuzzy Expert System depends upon the was developed using a self-organizing fuzzy rule-based system [2].

According to [12], Arthritis is a chronic disease and about three fourth of the patients are suffering from osteoarthritis and rheumatoid arthritis which are undiagnosed and the delay of detection may cause the severity of the disease at higher risk. A system for the diagnosis of Arthritis using fuzzy logic controller (FLC) was designed which was, a successful application of Zadeh's fuzzy set theory [16]. It is a potential tool for dealing with uncertainty and imprecision [12]. [10], designed a decision support system for malaria and dengue (DSSMD). The diagnosis of disease was solely based on the non - clinical symptoms of the disease using Artificial intelligence. In another study [6] developed a fuzzy expert system for the management of malaria (FESMM) which provides decision support platform to malaria researchers, physicians to assist malaria researchers, physicians and other health practitioners in malaria endemic regions.

Ethambutol (EMB). Each treatment regimen consists of an initial 2-month treatment phase followed by a continuation phase of either 4 or 7 months. The 4-month continuation phase is used for the majority of patients. Although these regimens are broadly applicable, there are modifications that should be made under specified circumstances.

In this section, we show the fuzzy expert system designed, membership functions fuzzification, fuzzy rule base and defuzzification.

opinion of the domain experts on various issues related to the field of study. The developed Fuzzy Expert System for the Management of tuberculosis has an architecture as shown in Figure 1 below. 


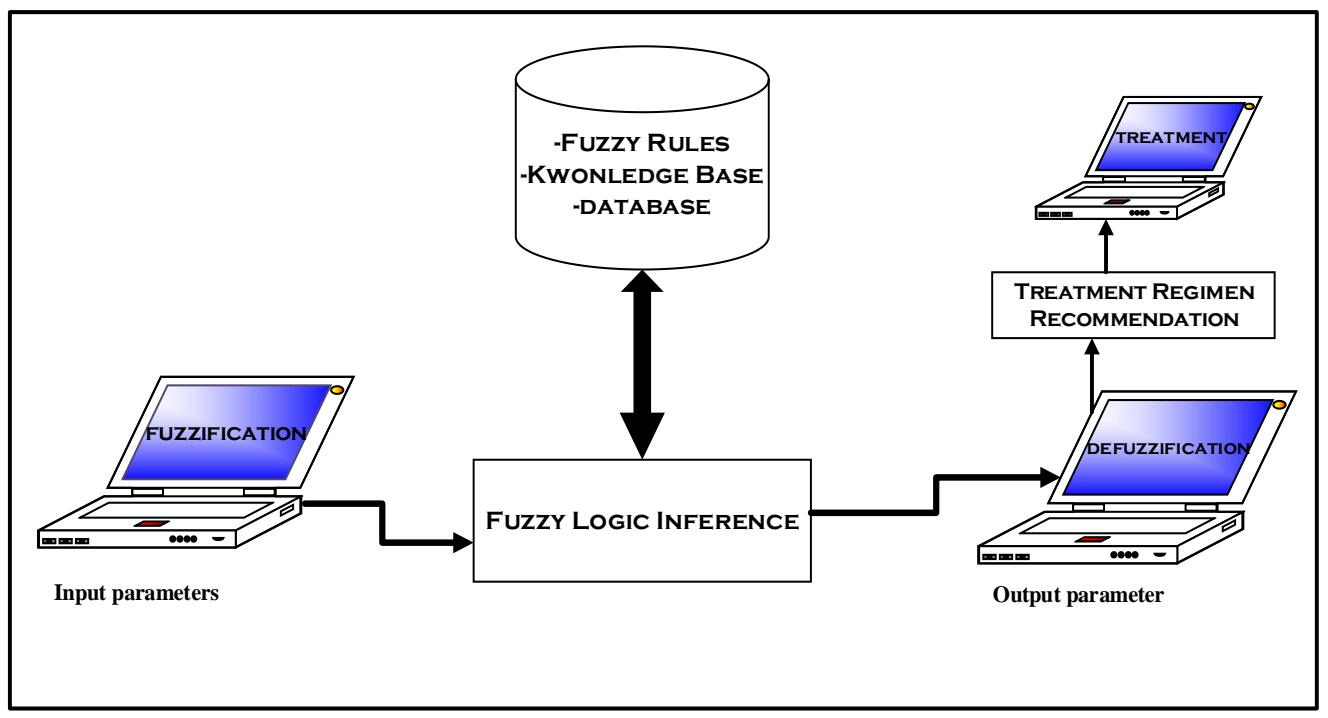

Figure 1: Architectural Design of the Proposed Diagnosis and Treatment of TB.

\subsection{Inputs/Output Membership Function \\ (Fuzzification/Defuzzification) for the Proposed System.}

In this section we defined fuzzy input parameters which are the symptoms of TB with their linguistic categories described as low, medium and high to be used for the diagnosis and treatment based on the classes of TB. The fuzzy input parameters (symptoms scores) we be fuzzified using triangular membership functions. The inputs for the proposed system are: Chest pain $(\mathrm{CP})$, cough duration (CD), fever duration (FV), night sweats (NS), weight loss (WL), loss of appetite (LOA), change in bowel habits $(\mathrm{CBH})$, variations in mental behavior (VMB), masses along the neck (MAN), draining sinus (DS), coma (seizure) (CO), stiff Neck (SN), headache (HD), abdominal Pain (AP), painful or uncomfortable urination (PU), hemopysis (coughing up blood) (CUB), fatigue (FA) and blood present in urine (BPU). This inputs parameters are model in a range of six weeks. The low level of the symptoms shows that TB is just beginning in the patient body, the medium stage indicate that the patient situation is becoming bad and the final stage which is the high level indicate that the patient case has gone to a very bad state, which if not carefully handled the patient might loss his/her life. This scenario is the same for all the TB symptoms to consider in this research work .Figures $3.2 \mathrm{a}$ and $3.2 \mathrm{~b}$ shows the membership function distribution of the inputs symptoms. 


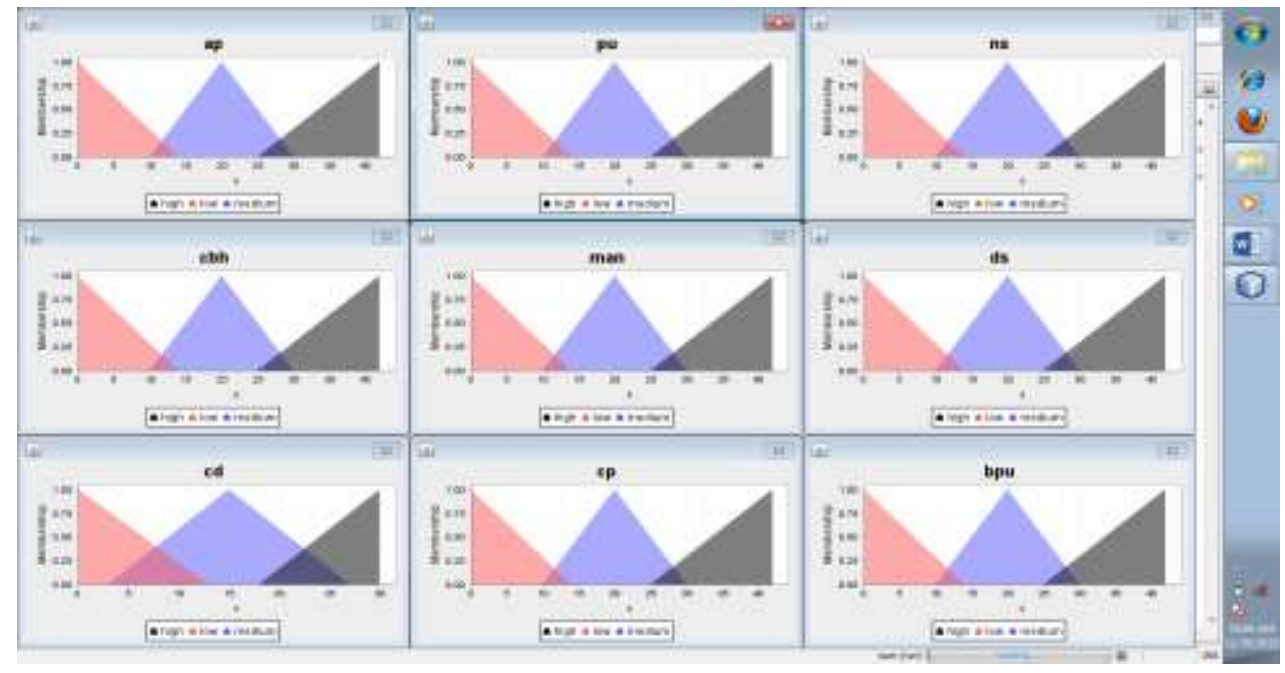

Figure 2a: membership function of the inputs variables

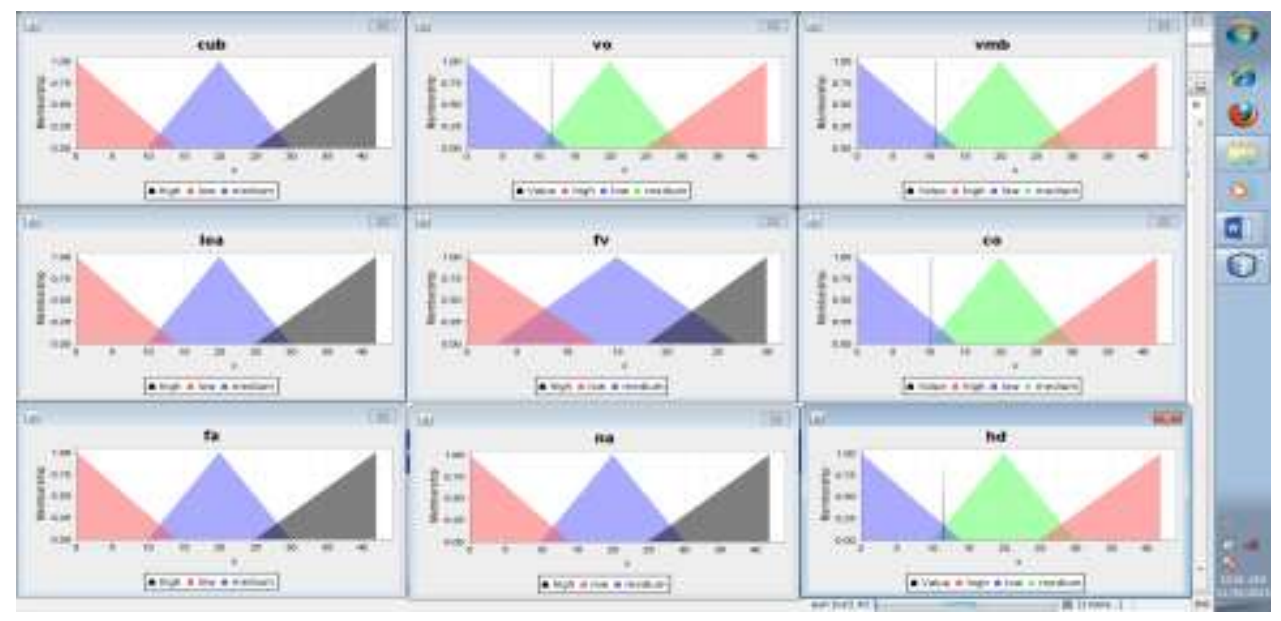

Figure $2 b$ : membership function of the inputs variable

\subsection{The Diagnosis/Output Design for System}

This subsection describes the output of the fuzzy logic engine. There are five output variables, we www.ijcat.com shall call them "Tuberculosis Group", namely: Tuberculosis meningitis (TBM), Gastrointestinal tuberculosis (GITB), Tuberculosis lymphadenitis (TBL), Genitourinary tuberculosis (GUTB) and 
Pulmonary tuberculosis (PTB). Here too the triangular membership functions will be used on each output variable. The membership functions details are shown in Table 1.

Table 1 The Output variable (Tuberculosis Group) ranges that correspond to each fuzzy set

\begin{tabular}{|l|l|l|}
\hline Output field & Range & Fuzzy Set \\
\hline & $0<\mathrm{TBG}<3$ & TBM1 \\
& $3<\mathrm{TBG}<7$ & TBM2 \\
& $7<\mathrm{TBG}<10$ & TBM3 \\
\cline { 2 - 3 } & $0<\mathrm{TBG}<3$ & GITB1 \\
& $3<\mathrm{TBG}<7$ & GITB2 \\
& $7<\mathrm{TBG}<10$ & GITB3 \\
\cline { 2 - 3 } TUBERCULOSIS GROUP (TBG) & $0<\mathrm{TBG}<3$ & TBL1 \\
& $3<\mathrm{TBG} 7$ & TBL2 \\
& $7<\mathrm{TBG}<10$ & TBL3 \\
\cline { 2 - 3 } & $0<\mathrm{TBG}<3$ & GUTB1 \\
& $3<\mathrm{TBG}<7$ & GUTB2 \\
& $7<\mathrm{TBG}<10$ & GUTB3 \\
\cline { 2 - 3 } & $0<\mathrm{TBG}<3$ & PTB1 \\
& $3<\mathrm{TBG}<7$ & PTB2 \\
& $7<\mathrm{TBG}<10$ & PTB3 \\
\hline
\end{tabular}

From table 1 it shows that, the higher the value, the higher the health risk of the patient. In this system, we have 15 fuzzy sets for the output variable risk group (TBM1, TBM2, TBM3, GITB1, GITB2, GITB3, TBL1, TBL2 TBL3, GUTB1, GUTB2, GUTB3, PTB1, PTB2 and PTB3). Each of the five output variable has three fuzzy set, which represent the low, medium and high level of TB disease to be diagnosed. To get a particular class of $\mathrm{TB}$, a set of inputs (symptoms) must be inputted into the system. This inputs will also identify the degree of risk of the patient whether the level of TB is low, medium or high. For example, to detect T BM1, TBM2 or TBM3. if the values of inputs are in the range of 0 to 3, then the level of TB is TBM1 which is low, also if the values of inputs are in the range of 3 to 7 , then the TB level is TBM2 which is medium and if the values inputted are in the range of 7 to 10 , then then level of TB is TBM3, which is high. The mode of evaluation is the same to other classes of TB to be diagnosed by the system. Figure 3 shows the membership functions of the output variables. 


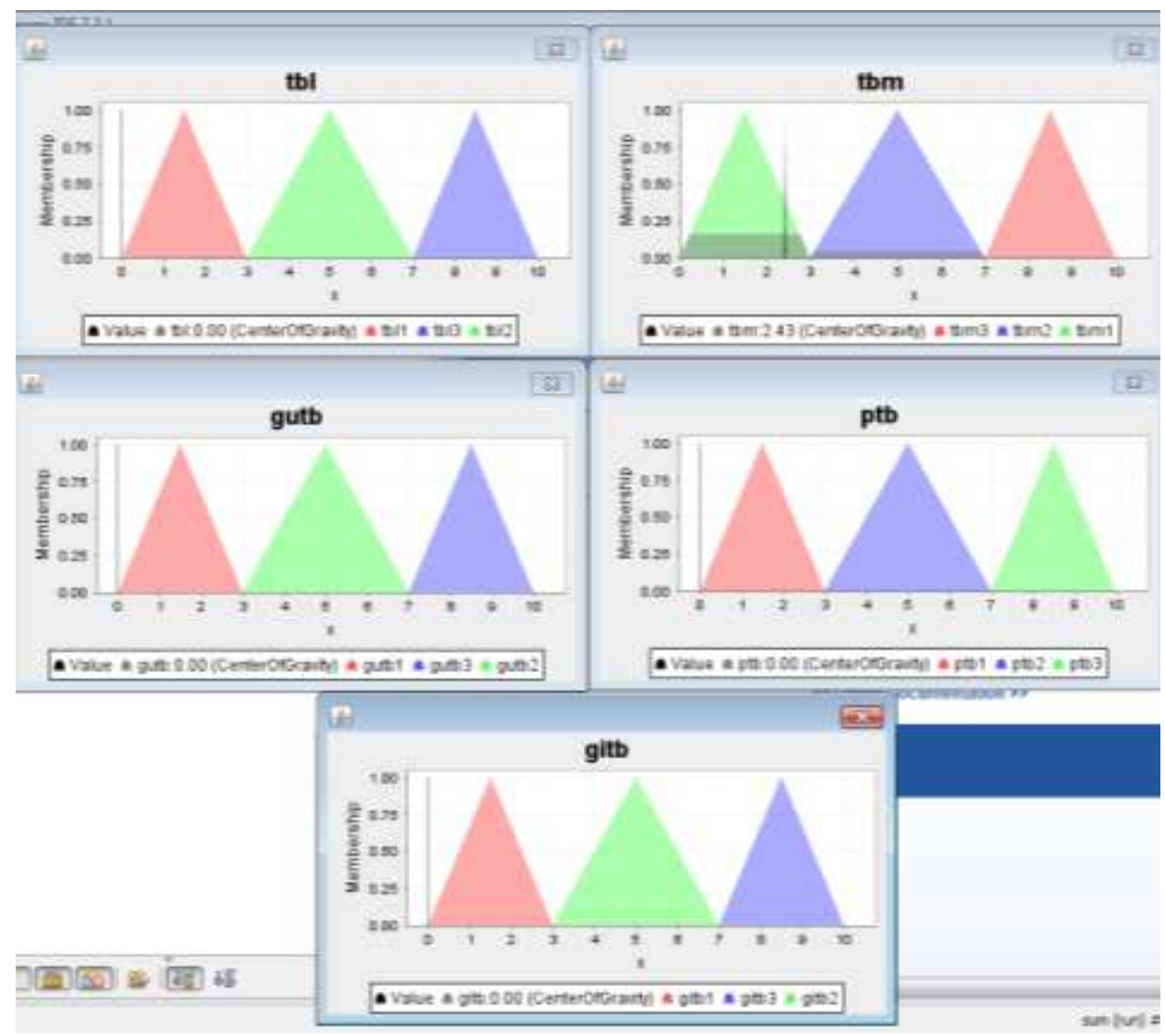

Figure 3: membership functions of the output (TBG) variables.

\subsection{Activity Diagram}

Activity diagram describes the business and operational step-by-step workflows of components in a system. It shows the overall flow of control detailing the sequence of activities from a start point to the finish point displaying the many decision paths that exist in the progression of events contained in the activity. They may be used to detail situations where parallel processing may occur in the execution of some activities. Activity diagram for the fuzzy-based system is shown in figure 4 . Before the doctor accesses the system, he/she must be authenticated. 

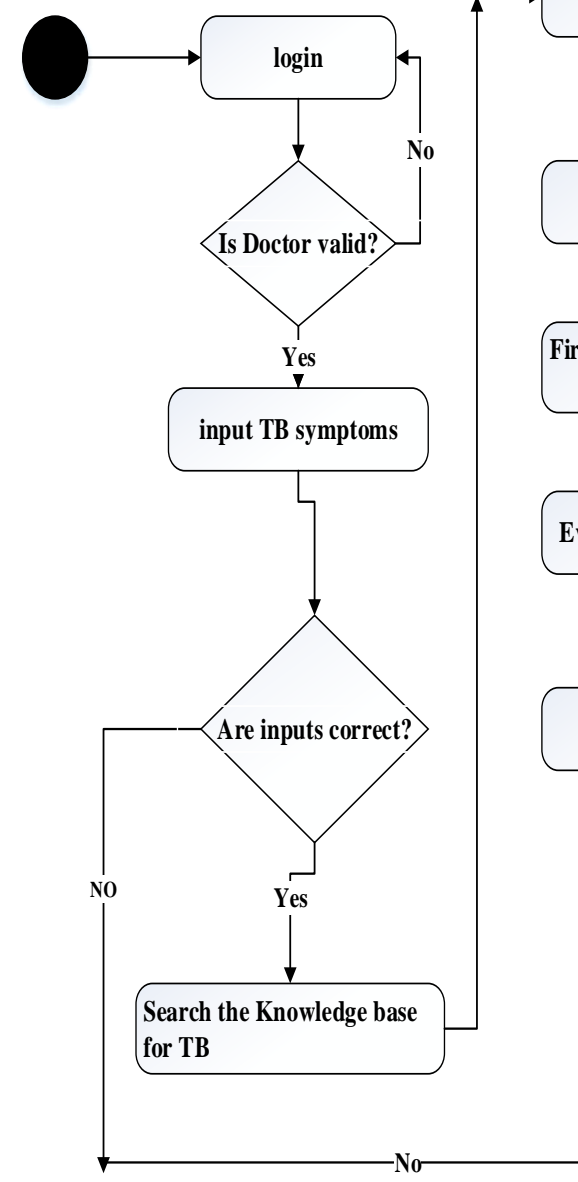

\section{Get the weighing factors}
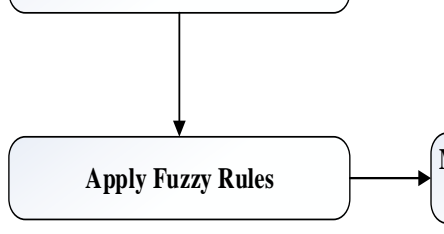

Map Fuzzy Inputs into their respective weighing factors

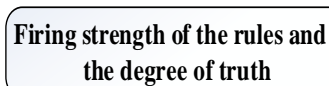

Evaluate the group/class of TB

\section{Select treatment regimen}

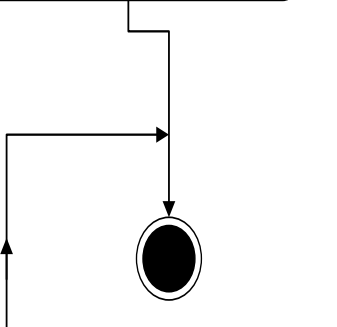

Figure 4: Activity Diagram for the Proposed Diagnosis and Treatment of TB.

\subsection{Defuzzification of the Outputs for the System}

The input for the defuzzification process is a fuzzy set (the aggregate output fuzzy set), and the output is a single number. As much as fuzziness helps the rule evaluation during the intermediate steps, the final desired output for each variable is generally a single number. However, the aggregate of a fuzzy set encompasses a range of

www.ijcat.com output values, and so they must be defuzzified in order to resolve a single output value from the set. Perhaps the most popular defuzzification method is the centroid or fuzzy centroid, this method returns the centre of an area under the curve. . It computes the defuzzified result by determining the element for which the combined set's area is divided into two equal faces (Klir and Yuan, 1995). This is the method adopted. The defuzzification of the data into a crisp output is accomplished using the "Fuzzy Centroid". 
Fuzzy Centroid Formula is as shown in equation 1.

Output $=\frac{\sum_{i=1}^{n}\left(\text { Center }_{i} * \text { Strength }_{i}\right)}{\sum_{i=1}^{n} \text { Strength }_{i}}$

\subsection{Fuzzy Inference Component for the System}

A fuzzy inference system is usually composed of one or more Function Blocks (FB). Each FB has variables (input, output or instance variables) as well as one or more Rule Blocks (RB). Each rule block is composed of a set of rules, as well as Aggregation, Activation and Accumulation methods. All methods defined in the norm are implemented in jFuzzyLogic. We will adhere to the definitions of Aggregation, Activation and Accumulation as defined by IEC-61131-7. Aggregation methods define the t-norms and tconorms playing the role of intersection, union and complement operators. Activation method define how rule antecedents modify rule consequents, i.e. once the IF part has been evaluated, how this result is applied to the THEN part of the rule. Some of the fuzzy rules for this work are as shown below.

\section{RULEBLOCK No1}

AND: MIN; // Use 'min' for 'and' (also implicit use

//'max' for 'or' to fulfill DeMorgan's Law)

ACT: MIN; // Use 'min' activation method

ACCU: MAX; // Use 'max' accumulation method

RULE 1: IF sn IS low AND hd IS low AND vmb IS low AND co IS low AND vo IS low THEN tbm IS tbm1;

RULE 2: IF sn IS high AND hd IS high AND vmb IS high AND co IS high AND vo IS high THEN tbm IS tbm3;

www.ijcat.com
Where $\mathrm{n}$, is the number of output members.

RULE 3: IF sn IS medium AND hd IS medium AND vmb IS medium AND co IS medium AND vo IS medium THEN tbm IS tbm2;

RULE 4: IF ap IS low AND fv IS low AND wl IS low AND cbh IS low AND vo IS low AND na IS low THEN gitb IS gitb1;

RULE 5: IF ap IS medium AND fv IS medium AND wl IS medium AND cbh IS medium AND vo IS medium AND na IS medium THEN gitb IS gitb2;

RULE 6: IF ap IS high AND fv IS high AND wl IS high AND cbh IS high AND vo IS high AND na IS high THEN gitb IS gitb3;

RULE 7: IF man IS low AND ds IS low THEN tbl IS tbl1;

RULE 8: IF man IS medium AND ds IS medium THEN tbl IS tbl2;

RULE 17: IF man IS high AND ds IS low THEN tbl IS tbl2;

RULE 97: IF man IS medium AND ds IS high THEN tbl IS tbl2;

RULE 98: IF man IS high AND ds IS medium THEN tbl IS tbl2;

RULE 99: IF man IS medium AND ds IS low THEN tbl IS tbl1;

RULE 100: IF man IS low AND ds IS medium THEN tbl IS tbl1;

END_RULEBLOCK

END_FUNCTION_BLOCK 


\subsection{Treatment Regimen for TB}

In the treatment of $\mathrm{TB}$ there are four basic treatment regimens recommended for treating patients with TB disease caused by organisms that are known. These drugs include Isoniazid (INH), Rifampin (RIF), Pyrazinamide (PZA),

\section{RESULTS}

and Ethambutol (EMB), etc. Each treatment regimen consists of an initial 2-month treatment phase followed by a continuation phase of either 4 or 7 months. The 4 -month continuation phase is used for the majority of patients.

The welcome/Login interface for the designed Fuzzy-based system for TB diagnosis is as shown in figure 5

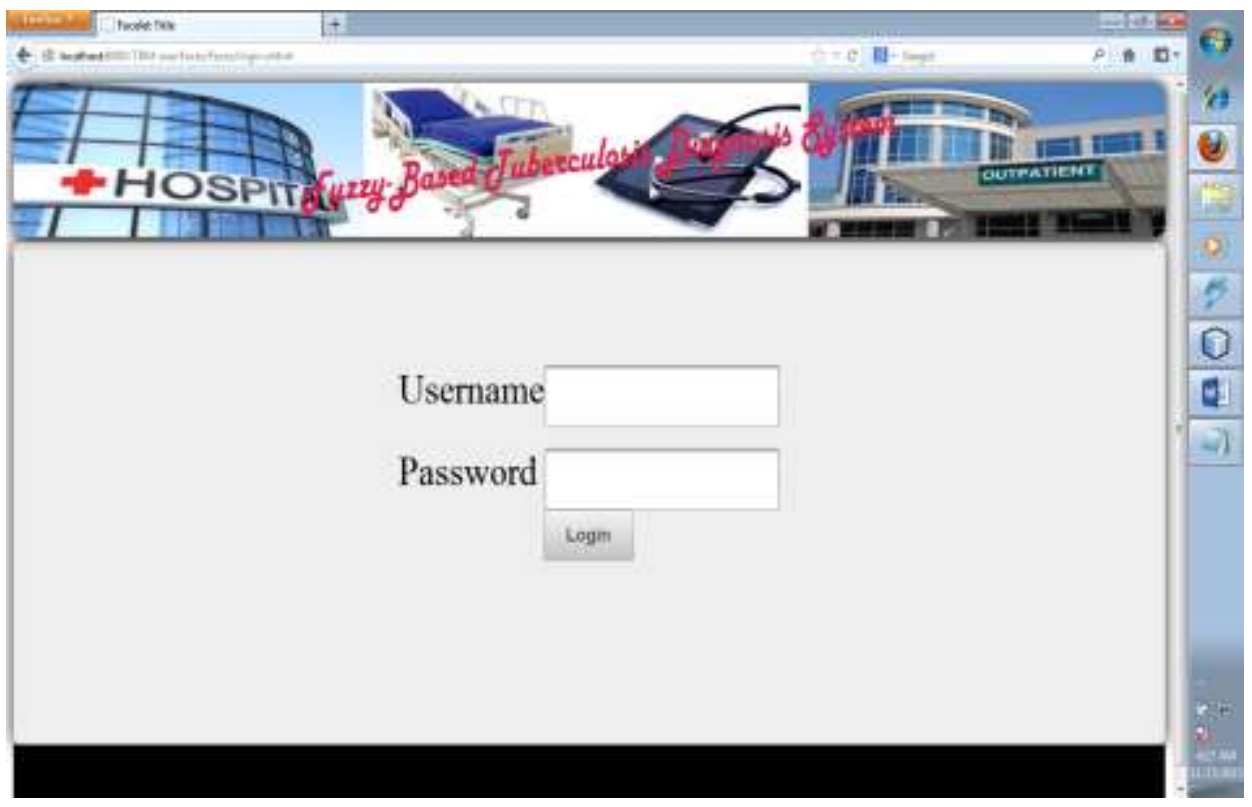

Figure 5: Login interface/welcome page.

Symptom selection interface for the designed Fuzzy-based system for TB diagnosis is as shown in figure 6 
International Journal of Computer Applications Technology and Research

Volume 5- Issue 2, 34 - 48, 2016, ISSN:- 2319-8656

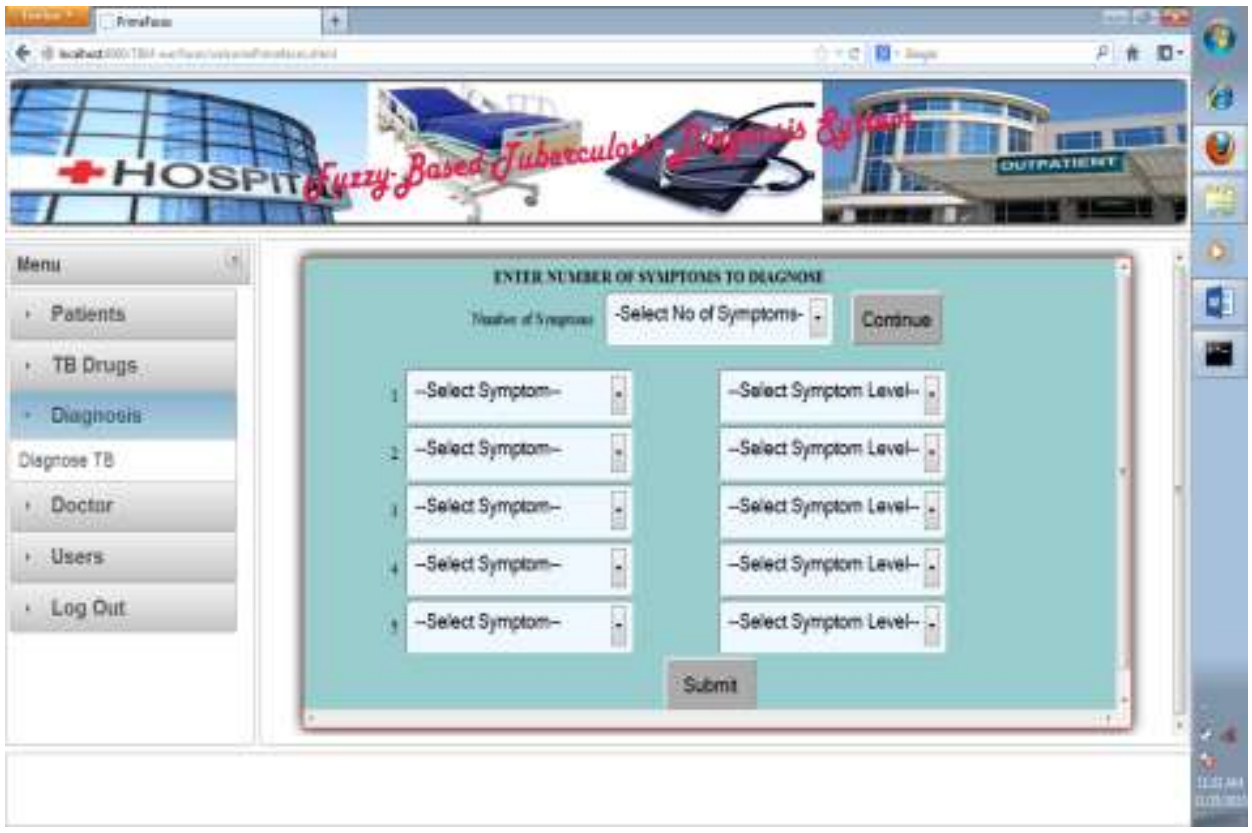

Figure 6: symptoms selection interface

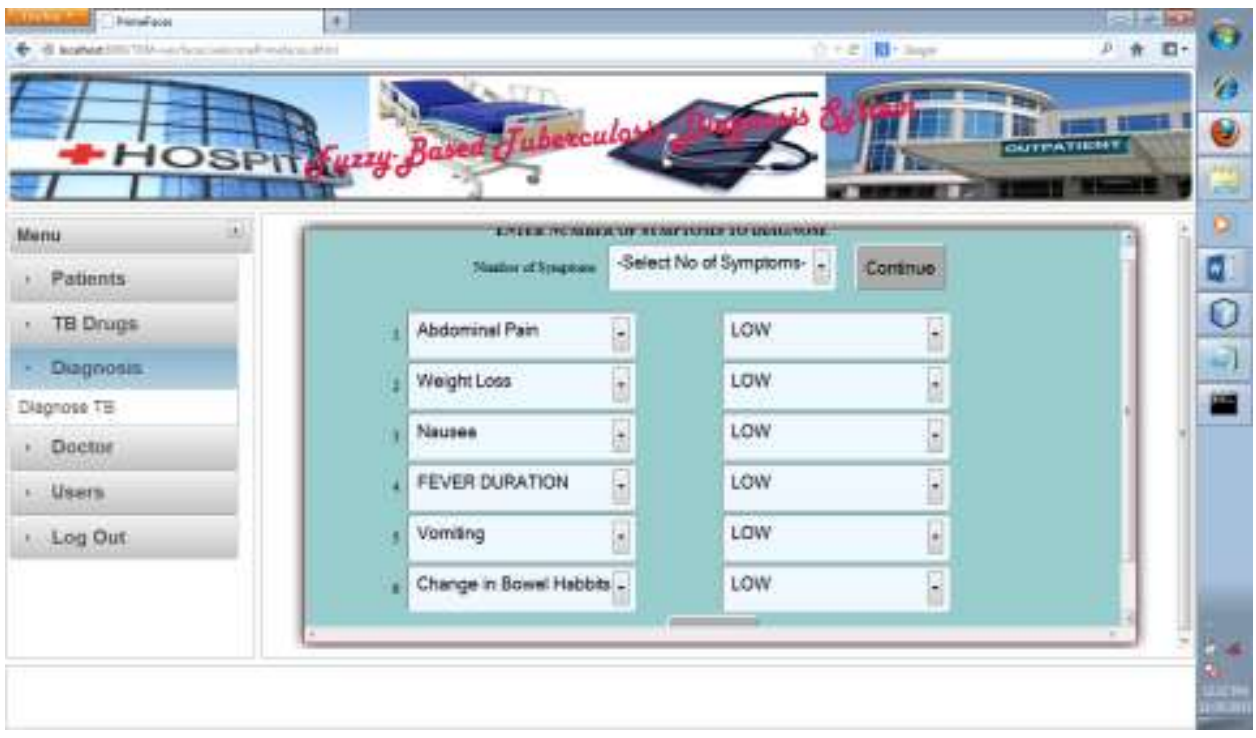

www.ijcat.com

Figure 7: Symptoms Selection for a particular Case.

wwijcat.com 
International Journal of Computer Applications Technology and Research

Volume 5- Issue 2, 34 - 48, 2016, ISSN:- 2319-8656

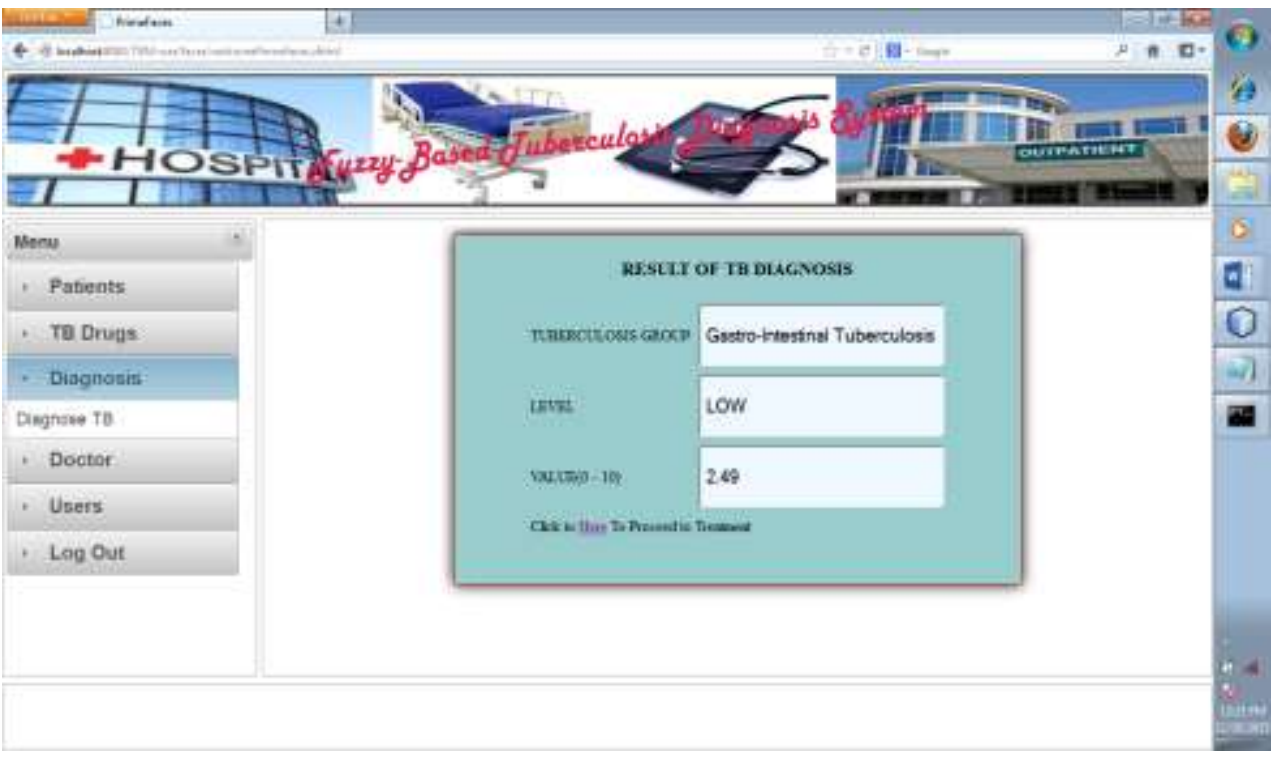

Figure 8: Diagnosed Result for Gastrointestinal TB

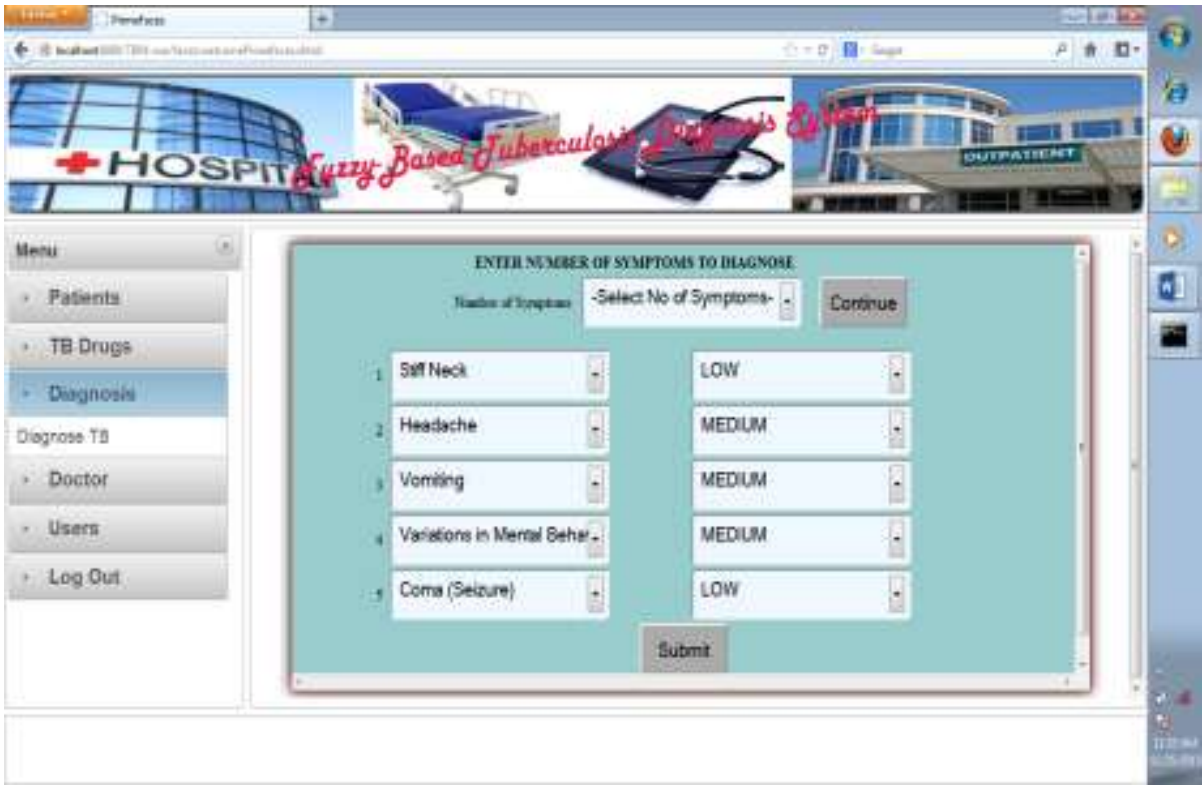

Figure 9: Five (5) Symptoms Selected for a particular Case. 
International Journal of Computer Applications Technology and Research

Volume 5- Issue 2, 34 - 48, 2016, ISSN:- 2319-8656

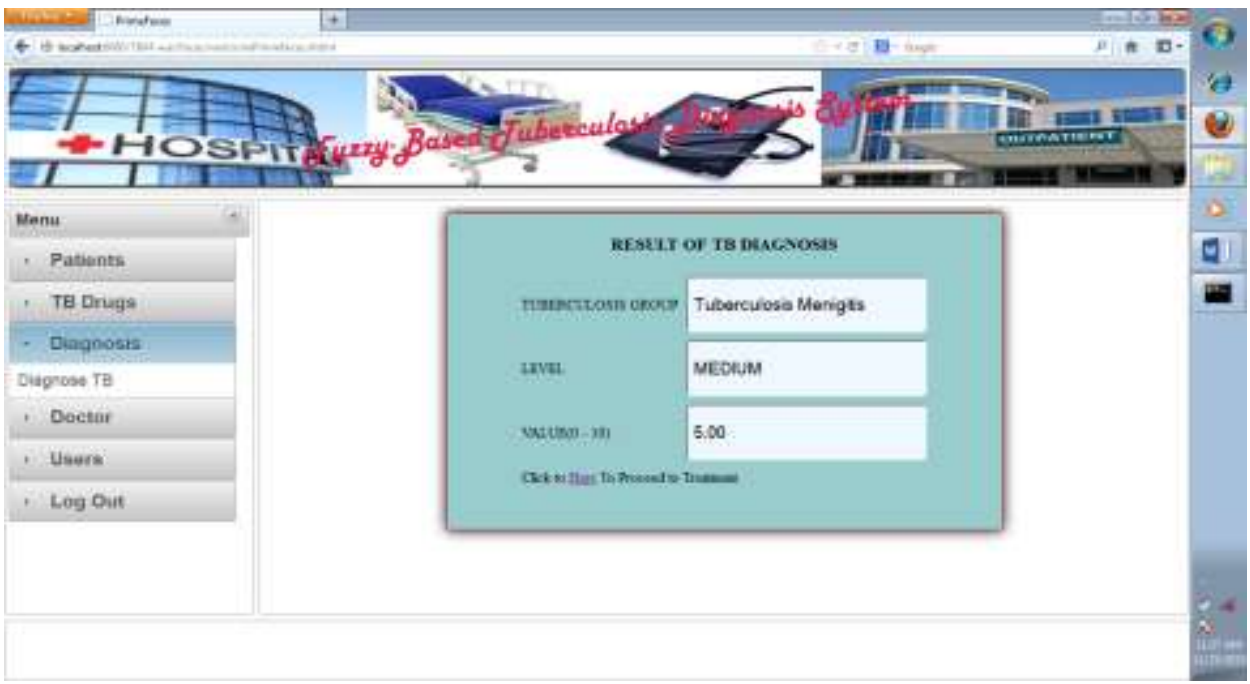

Figure 10: Diagnosed Result for Tuberculosis Menigitis

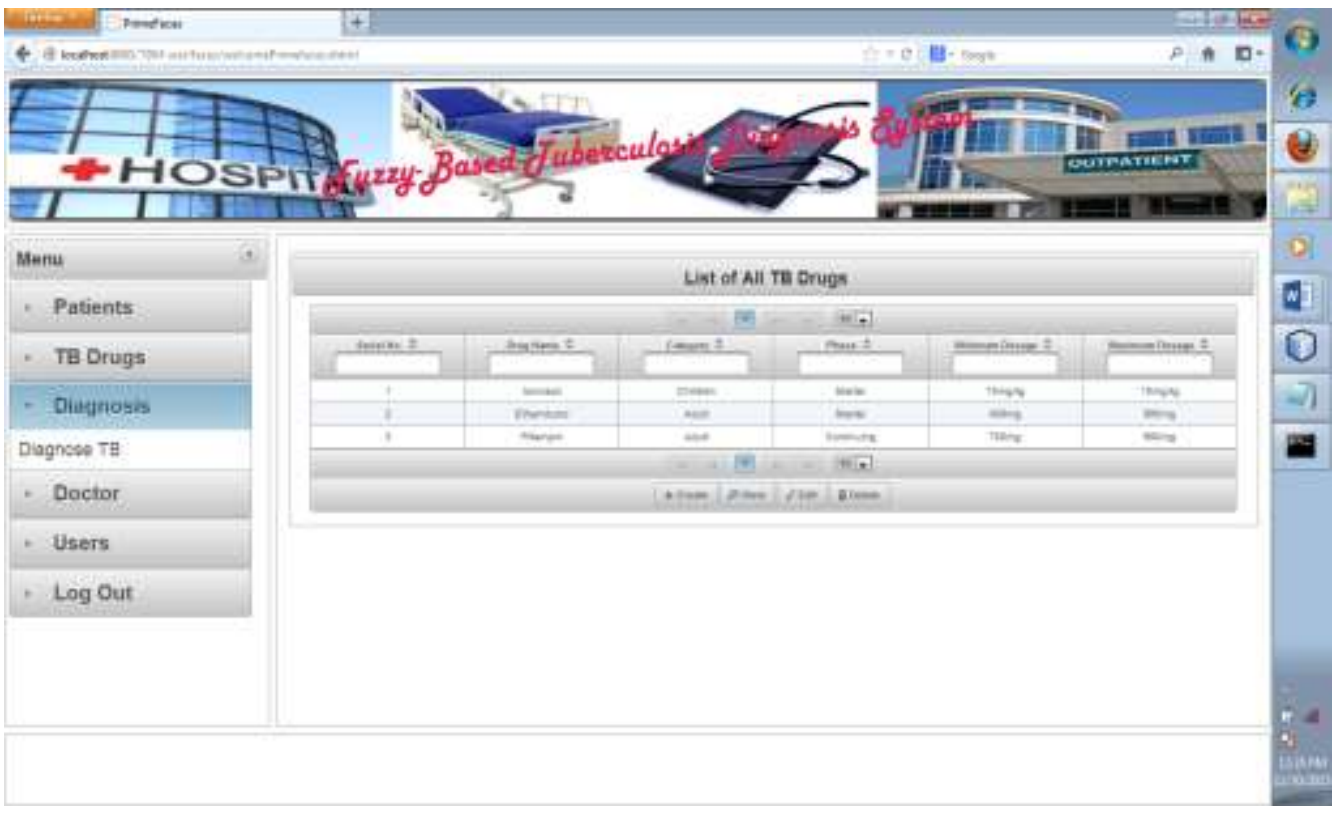

Figure 11: Drugs list Selections for Treatment

\section{DISCUSSIONS}

From figure 5, a user must be authenticated before he/she is allowed to use the system, on

www.ijcat.com gaining access into the system, the user (health personnel) will select the symptoms selections interface as shown in figure 6 , to fill in the 
symptoms as complained by the patient. Figure 7 shows a patient symptoms were selected and their intensities (abdominal pain was low, weight loss was low, nausea was low, fever duration was low, vomiting was low and change in bowel habit was low) upon diagnosis, the result was found to be Gastrointestinal TB with a degree risk value of 2.49 which is low and agreed with our design from table $1(0<\mathrm{TBG}<3)$. From figure 9 also another patient complained were selected with their degree of intensities (stiff neck was low, headache was medium, vomiting was medium, variation in metal behavior was medium and coma was medium) upon diagnosis the result was Tuberculosis Menigitis with a degree risk value of 5.00 as shown in figure 10 , which also agreed with our design from table $1(3<$ TBG $<7)$.

\section{REFERENCES}

[1] Abbas K., XuDe Z and Shaker K. (2010), Novel Respiratory Diseases Diagnosis by Using Fuzzy Logic. Global Journal of Computer Science and Technology Vol. 10 Issue 13 (Ver. 1.0).

[2] Ashish P, Jyotsna C, Shailendra K, Gupta M, VermaMembe K., Rajendra P, Qamar R, (2012), " Decision Support System for the Diagnosis of Asthma Severity Using Fuzzy Logic". Proceedings of the International MultiConference of Engineers and Computer Scientists 2012 Vol I, IMECS 2012, march 14-16, Hong Kong

[3] CDC (2013), Core Curriculum on Tuberculosis: What the Clinician Should Know. Centers for Disease Control and Prevention National Center for HIV/AIDS, Viral Hepatitis, STD, and TB Prevention Division of Tuberculosis Elimination. Retrieved from www.cdc.gov/tb.

[4] Chuen C. (1990), Fuzzy logic control system: Fuzzy logic controller -part I -, IEEE Transaction on systems, man, and cybernetics, Vol.20, No.2, pp.404 418.

[5] Djam, X.Y. and Y.H. Kimbi. (2011), A Decision Support System for Tuberculosis Diagnosis. Pacific Journal of Science and Technology. 12(2):410-425.
Figure 11 shows the list of drugs to be selected for treatment if the patient is affected with any of the TBG as designed in this research.

\section{CONCLUSION}

This Fuzzy-Based System for Tuberculosis Diagnosis and Treatment was designed with Java (JFuzzy Logic), membership functions, input variables, output variables and rule base. In this research there are 18 inputs or input variables and five outputs or output variables. The designed system has been tested with expert-doctor. This system is one of the simple and more efficient method for the diagnosis of $\mathrm{TB}$, within a short possible time.

For further work, we recommend that other diseases like cancer, be integrate into the system and also make it a mobile application.

[6] Djam X.Y., G. M. Wajiga, Y. H. Kimbi and N.V. Blamah, (2012) "A Fuzzy Expert System for the Management of Malaria",International Journal of Pure and Applied Sciences and Technology.

[7] Imianvan A.A. and Obi J.C. (2011), Fuzzy Cluster Means Expert System for the Diagnosis of Tuberculosis. Global Journal of Computer Science \& Technology Volume 11 Issue Version 1.0.

[8] Klir G. and Yuan B. (1995). Fuzzy Sets and Fuzzy Logic: Theory and Applications journal, 4(1), pp1-4.

[9] Kumar S and Kaur G (2013), Detection of Heart Diseases using Fuzzy Logic. International Journal of Engineering Trends and Technology (IJETT) - Volume 4 Issue 6.

[10] Priynka S, Singh D, Manoj K and Nidhi M (2013), Decision Support System for Malaria and Dengue Disease Diagnosis (DSSMD). International Journal of Information and Technology. Volume 3, Number 7, pp. 633-640. http://www. irphouse.com/ijict.htm.

[11] Raviglione, M. C. and O' Brien, R. J. (2004). Tuberculosis in Kasper DL., Braunwald E, Fauci As, Hauser SL, Longo DL Jameson JL, Isselbacher KJ, eds.: Hanison's Principles of Internal Medicine, 
$16^{\text {th }}$ ed., Mc Graw - Hill Professional, 953 $-66$.

[12] Singh S, Kumar A, Panneerselvam K and Vennila J. (2012), Diagnosis of arthritis through fuzzy inference system. Journal of Medical systems 2012 June

[13] Soundararajan K, Sureshkumar S and Anusuya C (2012), Diagnostics Decision Support System for Tuberculosis using Fuzzy Logic. International Journal of Computer Science and Information Technology \& Security (IJCSITS), Vol. 2, No.3.
[14] Shamshirband S, Somayeh H, Hossein J, Mohsen A, Shaghayegh V, Dalibor P, Abdullah G and Kiah L (2014), Tuberculosis Disease Diagnosis Using Artificial Immune Recognition System. International Journal of Medical Sciences 11(5): 508-514.

[15] William G. (2011). An Optimization Approach to Employee Scheduling Using Fuzzy Logic. (MSc. Thesis, California Polytechnic State University, San Luis Obispo).

[16] Zadeh L (1965), "Fuzzy Sets," Information and Control, Vol. 8, No. 3. 